\title{
Composição corporal e exigências nutricionais de cálcio e fósforo de cordeiros Santa Inês em crescimento(1)
}

\author{
Juan Ramón Olalquiaga Pérez(2), Luciana Castro Geraseev( ${ }^{(2)}$, Cristiane Leal dos Santos ${ }^{(2)}$, \\ Júlio César Teixeira ${ }^{(2)}$ e Sarita Bonagurio(2)
}

\begin{abstract}
Resumo - Dois experimentos foram conduzidos com o objetivo de determinar a composição corporal e estimar as exigências de Ca e P, para ganho em peso, de cordeiros Santa Inês em crescimento. Em cada experimento foram usados 18 animais com 25 a $35 \mathrm{~kg}$ de peso vivo (PV), no primeiro, e com 15 a $25 \mathrm{~kg}$ no segundo experimento. Seis animais foram abatidos no início de cada experimento para avaliar o conteúdo de Ca e P corporal, servindo como animais referência para o método do abate comparativo, seis animais receberam alimentação ad libitum e seis, alimentação restrita. As exigências líquidas de Ca e P para o ganho em peso foram estimadas a partir da derivação de equações de regressão do logaritmo da quantidade desses minerais presentes no corpo vazio, em função do peso do corpo vazio. As exigências líquidas por quilo de ganho de PV para animais com 15, 25 e $35 \mathrm{~kg}$ de PV foram, respectivamente: 11,63, 10,52 e $9,82 \mathrm{~g}$ de $\mathrm{Ca}$ e $5,82,4,99$ e 4,28 g de $\mathrm{P}$.
\end{abstract}

Termos para indexação: Ovis aries, ovinos, necessidade de nutrientes, nutrientes minerais.

Body composition and nutritional requirements of calcium and phosphorus in growing Santa Inês lambs

\begin{abstract}
Two experiments were conducted with the objective to determine body composition and to estimate the nutritional requirements in $\mathrm{Ca}$ and $\mathrm{P}$ in growing Santa Inês lambs. In each experiment, eighteen animals were used, with 25 to $35 \mathrm{~kg}$ of live weight, in the first, and with 15 to $25 \mathrm{~kg}$ in the second experiment. Six animals were slaughtered at the beginning of each experiment to access the amount of $\mathrm{Ca}$ and $\mathrm{P}$ retained in the body, which were used as reference animals for the comparative slaughter method. Six animals were fed ad libitum and six received restricted food. The net requirements of $\mathrm{Ca}$ and $\mathrm{P}$ for empty body weight gain were estimated from derivation in the regression equations of the total mineral present in the empty body based on the empty body weight. The net requirements of $\mathrm{Ca}$ and $\mathrm{P}$ for $\mathrm{kg}$ of live weight (LW) gain for lambs with 15, 25, and $35 \mathrm{~kg}$ of LW were, respectively, $11.63,10.52$ and $9.82 \mathrm{~g}$ of $\mathrm{Ca}$ and $5.82,4.99$ and $4.28 \mathrm{~g}$ of $\mathrm{P}$.
\end{abstract}

Index terms: Ovis aries, sheep, nutritional requirements, mineral nutrients.

\section{Introdução}

A ovinocultura no Brasil é hoje uma alternativa de exploração pecuária que vem alcançando um grande desenvolvimento, principalmente em relação à produção de carne. Nesse aspecto, a raça Santa Inês tem demonstrado ser promissora, pois apresenta características como precocidade, alto rendimento de carcaça e adaptação a diversas condições ambientais.

\footnotetext{
(1) Aceito para publicação em 20 de julho de 2000 .

(2) Universidade Federal de Lavras (UFLA), Dep. de Zootecnia, Caixa Postal 37, CEP 37200-000 Lavras, MG.

E-mail: jroperez@ufla.br, lgerasev@ufla.br, msccii@ufla.br, jcteixei@ufla.br,sasabona@ufla.br
}

O entrave no uso dessa raça, no entanto, tem sido constatado pela falta de informações, principalmente quanto à composição corporal e exigências nutricionais.

$\mathrm{O}$ arraçoamento desses animais tem sido baseado em recomendações para ovinos lanados que foram estabelecidas em regiões temperadas, porém, segundo Loosli \& Guedes (1976), existem dúvidas quanto à validade do uso de requerimentos estabelecidos nas regiões temperadas para animais em regiões tropicais. Embora os experimentos de alimentação possam ser usados para estimar as exigências de minerais, eles só prevalecem para as condições em que o experimento foi conduzido. Assim, muitos 
países têm desenvolvido tabelas de exigências nutricionais adaptadas às suas condições (Silva, 1996).

O Agricultural Research Council (1980) adotou um valor médio para o conteúdo corporal de $\mathrm{Ca}$ e $\mathrm{P}$, para ovinos em crescimento, de $11 \mathrm{~g}$ de $\mathrm{Ca}$ e $6 \mathrm{~g}$ de $\mathrm{P}$ por kg de peso corporal vazio (PCV) e considerou que a concentração desses minerais no ganho de peso é independente do peso do animal. Já Annenkov (1982) e Grace (1983) encontraram valores médios, para cordeiros em crescimento, de 10,2 e 10,5 g de Ca e 5,4 e 5,2 $\mathrm{g}$ de $\mathrm{P}$ por $\mathrm{kg}$ de ganho de peso corporal, respectivamente.

O Agricultural Research Council (1980) e o National Research Council (1985) admitem que os requerimentos líquidos de macroelementos minerais são constantes e independem do peso do animal. Nas recomendações de exigências, o National Research Council (1985) considerou um requerimento absoluto de $\mathrm{Ca}$ e $\mathrm{P}$ para cordeiros em crescimento de $183 \mathrm{mg}$ de $\mathrm{Ca}$ e $103 \mathrm{mg}$ de $\mathrm{P}$ por $\mathrm{kg}$ de PV/dia, enquanto o Agricultural Research Council (1980) estimou o requerimento como $11 \mathrm{~g}$ de $\mathrm{Ca}$ e $6 \mathrm{~g}$ de $\mathrm{P}$ porkg de PCV.

Já o Agricultural and Food Research Council (1991) adotou equações baseadas no crescimento ósseo para estimar as exigências de $\mathrm{Ca}$ e $\mathrm{P}$ e considerou que a deposição desses elementos no corpo decresce à medida que $\mathrm{o}$ animal torna-se adulto.

Annenkov (1982) apresentou uma Tabela de exigências de $\mathrm{Ca}$ e $\mathrm{P}$ para várias categorias. Para animais com $10 \mathrm{~kg}$ de peso vivo (PV) e um ganho diário de $100 \mathrm{~g}$, foi recomendada uma ingestão de $1,7 \mathrm{~g}$ de $\mathrm{Ca}$ e $1,1 \mathrm{~g}$ de $\mathrm{P} / \mathrm{dia}$; para um ganho de $200 \mathrm{~g}$, a recomendação passa a ser 3,3 $\mathrm{g}$ de $\mathrm{Ca}$ e $1,9 \mathrm{~g}$ de $\mathrm{P} / \mathrm{dia}$; para animais com $20 \mathrm{~kg}$ de peso vivo e com um ganho diário de $100 \mathrm{~g}$, a recomendação é de $3,0 \mathrm{~g}$ de $\mathrm{Ca}$ e 1,3 g de P/dia; para um ganho diário de $200 \mathrm{~g}$, recomenda-se 5,0 g de $\mathrm{Ca}$ e 2,0 $\mathrm{g}$ de $\mathrm{P} /$ dia.

$\mathrm{Na}$ literatura verifica-se que existe variação muito grande nos valores de composição corporal e, conseqüentemente, nas exigências em Ca e P de ovinos. Segundo Boin (1985), em função das diferenças entre os sistemas de produção, raças, alimentos e condições climáticas, é importante a realização de trabalhos com o objetivo de verificar as exigências minerais, os quais devem ser feitos regionalmente com os animais nas condições dos sistemas de produção usados.

O objetivo deste trabalho foi determinar a composição corporal e estimar as exigências de $\mathrm{Ca}$ e $\mathrm{P}$ em cordeiros Santa Inês em crescimento, criados nas condições do Sul do Estado de Minas Gerais.

\section{Material e Métodos}

O trabalho foi desenvolvido no setor de Ovinocultura do Departamento de Zootecnia da Universidade Federal de Lavras, em Lavras, MG. Foram conduzidos dois experimentos utilizando-se 18 cordeiros machos inteiros da raça Santa Inês em cada um. No primeiro experimento, foram avaliadas a composição corporal e as exigências nutricionais, em Ca e $\mathrm{P}$, de cordeiros na faixa de 25 a $35 \mathrm{~kg}$ de peso vivo (PV), e no segundo foram avaliados os mesmos parâmetros com cordeiros na faixa de 15 a $25 \mathrm{~kg}$ de PV.

Os animais foram desmamados com 40 dias de idade $\mathrm{e}$ confinados em grupo, até atingirem o peso inicial de $15 \mathrm{ou}$ $25 \mathrm{~kg}$. Durante esse período receberam alimentação à vontade, cuja constituição era a mesma da dieta experimental (Tabela 1).

Ao atingirem o peso experimental inicial, seis animais foram abatidos, servindo como animais referência. Os demais entraram no experimento aos pares, sendo um para alimentação ad libitum e o outro para alimentação restrita. Os animais da alimentação ad libitum receberam quantidades de alimento que permitiam uma sobra de $20 \%$ do total oferecido, enquanto os animais do grupo de alimentação restrita receberam quantidades para atender a uma ingestão diária de energia metabolizável, correspondendo à exigência de mantença, com acréscimo de $20 \%$, segundo recomendações do Agricultural Research Council (1980).

Quando o animal da alimentação ad libitum atingiu o peso predeterminado para o abate $(35 \mathrm{e} 25 \mathrm{~kg}$, no primeiro e segundo experimentos, respectivamente), foi abatido juntamente com o seu par da alimentação restrita.

Antes do abate, os animais foram submetidos a um jejum de 16 horas, com acesso a água. $\mathrm{O}$ abate foi feito por sangramento, pelo corte da carótida e jugular, e o sangue coletado, pesado e congelado para análises posteriores. Após a coleta do sangue, o conteúdo do trato digestivo, bexiga e vesícula biliar foi eliminado para determinação do peso do corpo vazio. O corpo do animal (subdividido em partes menores), juntamente com o aparelho digestivo, vísceras, sangue, cabeça, patas e pele foram acondicionados em sacos de plástico, congelados, posteriormente moídos e amostrados para as análises químicas. $\mathrm{O}$ abate dos animais do grupo referência seguiu os mesmos procedimentos. 
Tabela 1. Composição da dieta experimental, expressa em porcentagem da matéria seca de ovinos da raça Santa Inês ${ }^{(1)}$.

\begin{tabular}{lcccccccc}
\hline Ingredientes & $\begin{array}{c}\mathrm{MS} \\
(\%)\end{array}$ & $\begin{array}{c}\mathrm{EM}^{(2)} \\
(\mathrm{kcal} / \mathrm{kg})\end{array}$ & $\begin{array}{c}\mathrm{PB} \\
(\%)\end{array}$ & $\begin{array}{c}\mathrm{Ca} \\
(\%)\end{array}$ & $\begin{array}{c}\mathrm{P} \\
(\%)\end{array}$ & $\begin{array}{c}\mathrm{Na} \\
(\%)\end{array}$ & $\begin{array}{c}\mathrm{Mg} \\
(\%)\end{array}$ & $\begin{array}{c}\mathrm{K} \\
(\%)\end{array}$ \\
\hline Milho (grão) & 66,23 & 2,087 & 6,49 & 0,022 & 0,196 & 0,023 & 0,060 & 0,230 \\
Farelo de soja & 12,37 & 0,394 & 6,28 & 0,053 & 0,097 & 0,005 & 0,030 & 0,223 \\
Feno coast cross & 20,25 & 0,395 & 2,44 & 0,120 & 0,080 & 0,008 & 0,041 & 0,362 \\
Calcário & 0,85 & - & - & 0,306 & - & - & - & - \\
Sal comum $_{\text {Sup. mineral }}^{(3)}$ & 0,25 & - & - & - & - & 0,092 & - & - \\
Sup. vitamínico $^{(3)}$ & 0,01 & - & - & - & - & - & - & - \\
\hline Total & 0,04 & - & - & - & - & - & - & - \\
\hline
\end{tabular}

(1)MS: matéria seca; EM: energia metabolizável; PB: proteína bruta. ${ }^{(2)}$ National Research Council (1985). ${ }^{(3)}$ Suplemento mineral e vitamínico (nutriente/ kg de suplemento): Vit. A, 2.500.000 UI; Vit. D3, 500.000 UI; Vit. E, $3.000 \mathrm{mg}$; tiamina, $750 \mathrm{mg}$; riboflavina, $1.000 \mathrm{mg}$; Vit. B12, $2.800 \mathrm{mcg}$; niacina, $500 \mathrm{mg}$; selênio, $150 \mathrm{mg}$; iodo, $1.000 \mathrm{mg}$; cobalto, $600 \mathrm{mg}$; ferro, $35.000 \mathrm{mg}$; cobre, $20.000 \mathrm{mg}$; manganês, $49.000 \mathrm{mg}$; zinco, $75.000 \mathrm{mg}$.

As análises químicas foram efetuadas segundo metodologia descrita por Silva (1981). As análises para determinação do Ca e P na matéria seca desengordurada do corpo do animal foram efetuadas por meio da digestão ácida com ácido nítrico e ácido perclórico, obtendo-se, desta forma, a solução mineral, a partir da qual foram feitas diluições para determinação do Ca e P.

O P foi determinado por redução do complexo fósforomolibdato com ácido ascórbico e as leituras foram tomadas em colorímetro. Para o $\mathrm{Ca}$, as diluições foram feitas adicionando-se cloreto de estrôncio e as leituras tomadas em espectrofotômetro de absorção atômica.

Para predição do conteúdo de Ca e P por quilo de PCV dos animais, adotou-se o modelo exponencial $\mathrm{y}=\mathrm{ax}^{\mathrm{b}}$, preconizado pelo Agricultural Research Council (1980). O modelo foi logaritmizado conforme a equação que se segue:

$\log y=a+b \log x$

onde:

$\log \mathrm{y}=\operatorname{logaritmo}$ do conteúdo total de Ca ou P no corpo vazio;

$\mathrm{a}=$ intercepto;

$\log \mathrm{x}=\log$ aritmo do peso corporal vazio;

$\mathrm{b}=$ coeficiente de regressão do conteúdo do $\mathrm{Ca}$ ou $\mathrm{P}$ em função do peso corporal vazio (PCV).

Para composição do ganho em peso, utilizou-se a técnica do abate comparativo descrita pelo Agricultural Research Council (1980), a qual possibilita a determinação a partir da diferença entre o total de cada mineral retido no corpo vazio dos animais abatidos com 35 e $25 \mathrm{~kg}$ de peso vivo em relação ao total de cada mineral retido no corpo vazio dos animais referência.

Após a obtenção das equações de predição da composição corporal, foi realizada uma análise de comparação de equações lineares, segundo Snedecor \& Cochran (1967), entre as equações de predição obtidas com os animais de 15 a $25 \mathrm{~kg}$ de peso vivo e as obtidas com os animais de 25 a $35 \mathrm{~kg}$ de peso vivo. Essa análise compara os coeficientes de elevação, coeficientes de regressão e variâncias residuais de duas equações lineares com o objetivo de verificar a similaridade entre elas.

As exigências líquidas para o ganho em peso corporal vazio de $\mathrm{Ca}$ e $\mathrm{P}$ foram estimadas a partir da derivação das equações de composição corporal, obtendo-se equações do tipo $y^{\prime}=$ b. $10^{-a} \cdot P_{C V}{ }^{(b-1)}$. As exigências líquidas desses minerais para o ganho em peso vivo foram obtidas pela conversão do peso corporal vazio em peso vivo utilizando-se o fator 1,10 obtido pelo quociente PV/PCV dos animais. Os requerimentos dietéticos foram então estimados aplicando-se o método fatorial, segundo metodologia recomendada pelo Agricultural Research Council (1980).

\section{Resultados e Discussão}

\section{Composição corporal}

Na Tabela 2 são apresentados os resultados médios e respectivos desvios-padrão da composição corporal em $\mathrm{Ca}$ e $\mathrm{P}$ e peso dos animais estudados. Observa-se que, tanto para animais na faixa de 15 a $25 \mathrm{~kg}$ quanto para animais na faixa de 25 a $35 \mathrm{~kg}$ de $\mathrm{PV}$, ocorreu um aumento na concentração de matéria seca e gordura corporal em função do aumento do PV. Essa relação entre a concentração de gordura, matéria seca corporal e peso corporal foi observada por vários autores, entre outros, Burton \& Reid (1969), Rattray et al. (1973) e Searle et al. (1979). 
Tabela 2. Peso vivo e composição do corpo vazio dos animais de referência, e de alimentação restrita e ad libitum, em ovinos.

\begin{tabular}{lccc}
\hline Item & Referência & Restrita & Ad libitum \\
\hline \multicolumn{3}{c}{ Animais de 15 a 25 kg de peso vivo } \\
Peso vivo (kg) & $15,02 \pm 0,614$ & $14,50 \pm 0,774$ & $24,92 \pm 0,664$ \\
Peso de corpo vazio (kg) & $12,90 \pm 1,143$ & $12,65 \pm 1,367$ & $21,91 \pm 1,384$ \\
Matéria seca (\%) & $32,36 \pm 3,034$ & $34,53 \pm 1,580$ & $35,91 \pm 1,227$ \\
Proteína (\% M Nat) & $17,52 \pm 0,736$ & $17,93 \pm 0,738$ & $17,14 \pm 0,942$ \\
Gordura (\% M Nat) & $10,02 \pm 3,084$ & $12,17 \pm 1,506$ & $14,43 \pm 0,985$ \\
Energia (kcal/kg M Nat) & $2184 \pm 402$ & $2340 \pm 241$ & $2519 \pm 36$ \\
Cálcio (\% M Nat) & $1,520 \pm 0,062$ & $1,589 \pm 0,097$ & $1,396 \pm 0,160$ \\
Fósforo (\% M Nat) & $0,861 \pm 0,044$ & $0,899 \pm 0,034$ & $0,750 \pm 0,050$ \\
\hline & & Animais de 25 a $35 \mathrm{~kg}$ de peso vivo \\
Peso vivo (kg) & $24,31 \pm 0,976$ & $26,52 \pm 1,763$ & $34,80 \pm 0,748$ \\
Peso de corpo vazio (kg) & $20,60 \pm 1,308$ & $23,18 \pm 1,678$ & $30,06 \pm 1,095$ \\
Matéria seca (\%) & $36,84 \pm 2,696$ & $39,60 \pm 1,390$ & $40,15 \pm 2,668$ \\
Proteína (\% M Nat) & $19,24 \pm 1,962$ & $18,38 \pm 0,957$ & $18,20 \pm 0,909$ \\
Gordura (\% M Nat) & $13,86 \pm 2,806$ & $17,17 \pm 1,168$ & $18,62 \pm 2,180$ \\
Energia (kcal/kg M Nat) & $2328 \pm 285$ & $2757 \pm 226$ & $2714 \pm 295$ \\
Cálcio (\% M Nat) & $1,457 \pm 0,256$ & $1,402 \pm 0,069$ & $1,320 \pm 0,067$ \\
Fósforo (\% M Nat) & $0,691 \pm 0,077$ & $0,667 \pm 0,054$ & $0,617 \pm 0,026$ \\
\hline
\end{tabular}

Os valores de matéria seca encontrados neste trabalho (Tabela 2) foram menores que os obtidos por Burton et al. (1974) e Searle et al. (1979), e maiores que os propostos por Kellaway (1973) e Searle et al. (1982). Por sua vez, estão próximos aos valores de concentração de gordura estimados pelo Agricultural Research Council (1980), cuja concentração foi 8,4; 13,9 e 19,3\% do PCV, para cordeiros inteiros e não merinos, com 15, 25 e $35 \mathrm{~kg}$ de $\mathrm{PCV}$, respectivamente. Essas diferenças, principalmente nos valores de concentração de gordura, podem ser atribuídas às diferenças existentes entre as raças dos animais estudados, sexo, manejo alimentar e condições climáticas.

Quanto a concentração corporal de $\mathrm{Ca}$ e $\mathrm{P}$, os dados obtidos nos dois experimentos demonstraram diminuição na concentração desses minerais em função do aumento do PV dos animais (Tabela 2). Esse decréscimo nas concentrações de $\mathrm{Ca}$ e $\mathrm{P}$ pode ser explicado pelo aumento na concentração de gordura nos animais mais pesados e pela redução no crescimento ósseo à medida que aumenta o peso corporal, pois a maior parte do Ca e P corporal está presente nesse tecido. Segundo Nour \& Thonney (1987), existe uma relação inversa entre as concentrações minerais nos tecidos ósseos e comestíveis e a concentração de gordura corporal, uma vez que a gordura tem baixo teor de minerais, e portanto dilui esses elementos. $\mathrm{O}$ decréscimo no conteúdo de $\mathrm{Ca}$ e $\mathrm{P}$ por unidade de peso em função do aumento do peso corporal também foi observado em outras espécies, como bovinos (Carvalho, 1989; Lana et al., 1992; Pires et al., 1993; Estrada, 1996; Paulino, 1996) e caprinos (Resende, 1989; Ribeiro, 1995).

A partir do PV, PCV e quantidades corporais de Ca e P dos animais estudados, foram determinadas equações de regressão para estimar o PCV em função do PV, e para estimar as quantidades de $\mathrm{Ca}$ e $\mathrm{P}$ presentes no corpo vazio em função do PCV (Tabela 3). A análise de comparação entre as equações de predição da composição corporal em Ca para animais de 15 a $25 \mathrm{~kg}$ de PV (equação 1) e para animais de 25 a $35 \mathrm{~kg}$ de PV (equação 2) demonstrou não haver diferenças significativas para o intercepto, coeficiente de elevação e variância residual. Tal resultado indica que as equações são similares, e portanto deve-se utilizar uma equação geral, para a predição da composição corporal em Ca para animais 
Tabela 3. Equações de regressão para o peso de corpo vazio (PCV), em função do peso vivo $(\mathrm{g})$, e para a quantidade de cálcio e fósforo presentes no corpo vazio em função do PCV, em ovinos de raça Santa Inês.

\begin{tabular}{|c|c|c|}
\hline Item & Equação & $\mathrm{R}^{2}(\%)$ \\
\hline & \multicolumn{2}{|l|}{ Animais de 15 a $25 \mathrm{~kg}$ de peso vivo } \\
\hline Peso $(\mathrm{g})$ & $\mathrm{PCV}=-671,20+0,9089 \mathrm{PV}$ & 96,68 \\
\hline Fósforo $(\mathrm{g})$ & $\log P=-0,91433+0,721648 \log$ & 92,12 \\
\hline Cálcio $(\mathrm{g})$ & $\log C a=-1,04340+0,813209 \log P C V$ & 88,61 \\
\hline & \multicolumn{2}{|l|}{ Animais de 25 a $35 \mathrm{~kg}$ de peso vivo } \\
\hline Pes & & 96,82 \\
\hline Fósfe & $\log P=-1$, & 73,06 \\
\hline Cálcio $(\mathrm{g})$ & $\log \mathrm{Ca}=-0,90659+0,782850 \log P C V$ & 60,19 \\
\hline \multicolumn{3}{|c|}{ Gera } \\
\hline $\begin{array}{l}\text { Fósforo }(\mathrm{g}) \\
\text { Cálcio }(\mathrm{g})\end{array}$ & $\begin{array}{l}\log P=-0,429811+0,602921 \log P C V \\
\log C a=-1,06474+0,818621 \log P C V\end{array}$ & \\
\hline
\end{tabular}

com PV na faixa de 15 aos $35 \mathrm{~kg}$. No caso do P, a análise de comparação entre as equações de predição da composição corporal para animais de 15 a $25 \mathrm{~kg}$ de PV (equação 1) e para animais de 25 a $35 \mathrm{~kg}$ de PV (equação 2) demonstrou não haver diferenças significativas para o intercepto e variância residual, entretanto houve diferença entre os coeficientes de elevação das duas equações. Esse resultado indica que as equações não são similares, e portanto não seria possível a utilização de uma equação geral, para a predição da composição corporal em $\mathrm{P}$ para animais com PV na faixa de 15 aos $35 \mathrm{~kg}$.

A partir das equações listadas na Tabela 3, estimou-se a composição em Ca e P do corpo vazio dos animais em função do PCV (Tabela 4).

Para animais na faixa de 15 a $25 \mathrm{~kg}$ de PV, a concentração de Ca no corpo vazio dos animais estimada a partir da equação geral variou de 15,477 a $14,143 \mathrm{~g} / \mathrm{kg}$ de PCV. Esses valores são semelhantes aos estimados utilizando-se uma equação parcial, na qual eles variaram de 15,431 a 13,973 g/ $\mathrm{kg}$ de PCV (Geraseev et al., 2000).

Assim como ocorreu com a composição corporal, os valores estimados da concentração corporal de Ca e P também diminuíram em função do aumento no peso vivo (Tabela 4). Annenkov (1982) também encontrou valores de concentração corporal de Ca e P decrescentes para cordeiros na faixa de 10 a $30 \mathrm{~kg}$ de $\mathrm{PV}$, sendo que o conteúdo de $\mathrm{Ca} / \mathrm{kg}$ de $\mathrm{PCV}$ variou de 10,3 a 10,2 g e o de fósforo variou de 6,4 a 5,7 g de $\mathrm{P} / \mathrm{kg}$ de PCV.
Como já discutido anteriormente, esse decréscimo na concentração corporal de $\mathrm{Ca}$ e $\mathrm{P}$ é devido, principalmente, à redução no crescimento ósseo à medida que o animal aumenta o peso corporal e se aproxima do peso adulto do grupo genético ao qual pertence.

Entretanto, os resultados encontrados nesta pesquisa diferem dos valores propostos pelo Agricultural Research Council (1980), que considera a deposição de $\mathrm{Ca}$ e $\mathrm{P}$ constante, independente do peso do animal, e estima um valor de $11 \mathrm{~g}$ de Ca e $6 \mathrm{~g}$ de $\mathrm{P} / \mathrm{kg}$ de PCV. Porém, em 1991, o Agricultural and Food Research Council reexaminou o modelo proposto pelo Agricultural Research Council (1980) e admitiu que o depósito de $\mathrm{Ca}$ e $\mathrm{P}$ decresce com a maturidade, o que confirma os resultados encontrados nesta pesquisa.

As diferenças nas concentrações de $\mathrm{Ca}$ e $\mathrm{P}$ corporais são reflexo, principalmente, das diferenças existentes na proporção de ossos na carcaça, uma vez que, aproximadamente, $99 \%$ do conteúdo de $\mathrm{Ca}$ e $80 \%$ do conteúdo de $\mathrm{P}$ no corpo estão nos ossos. Outro fator que explica as diferenças nas concentrações desses minerais é a variação na concentração de gordura, sendo esta função da idade, raça, grupo genético, sexo, manejo alimentar e condições climáticas aos quais o animal encontra-se submetido.

Desta forma, os valores de composição corporal propostos pelo Agricultural Research Council (1980), National Research Council (1985) e Agricultural and Food Research Council (1991) não devem simplesmente ser extrapolados, principalmente no caso de ovinos Santa Inês, uma vez que os resultados não são aplicáveis em razão das diferenças citadas anteriormente.

\section{Composição do ganho em peso}

A partir da derivação das equações de predição da composição corporal de Ca e P (Tabela 3), foram obtidas as equações que permitiram estimar as quantidades de $\mathrm{Ca}$ e $\mathrm{P}$ depositadas por quilograma de ganho em PCV, nas diferentes faixas de peso (Tabela 5). A concentração de Ca no ganho de PCV encontrada foi de 12,74 a $10,87 \mathrm{~g} / \mathrm{kg}$ de $\mathrm{PCV}$ e de $\mathrm{P}$ foi de 6,36 a 4,76 g/kg de PCV para animais com 12,5 a $30 \mathrm{~kg}$ de PCV, respectivamente. Por serem as quantidades de Ca e P no ganho de PCV reflexo da com- 
posição corporal, os valores encontrados nesta pesquisa para a composição do ganho diferiram dos valores preconizados pelo Agricultural Research Council (1980) e pelo National Research Council (1985).

Para a composição do ganho, o Agricultural and Food Research Council (1991) reexaminou e modificou o modelo proposto pelo Agricultural Research Council (1980), introduzindo o peso à maturidade no cálculo, considerando, desta forma, o decréscimo na deposição desses minerais com o avanço da maturidade. Encontraram para animais de 15 e $35 \mathrm{~kg}$ de PV os valores 11,48 e 9,06 g de Ca/PV, e 6,62 e 5,48 g de $\mathrm{P} / \mathrm{kg} \mathrm{PV}$, respectivamente.

Da mesma forma que para a composição corporal, as diferenças na quantidade de $\mathrm{Ca}$ e $\mathrm{P}$ do ganho são reflexos, principalmente, das diferenças existentes na proporção de ossos e de gordura na carcaça.

\section{Exigências de Ca e $P$}

As exigências líquidas de $\mathrm{Ca}$ e $\mathrm{P}$ para o ganho de PV foram calculadas dividindo-se as exigências líquidas para o ganho de PCV (Tabela 5) pelo fator 1,10 calculado a partir das equações de conversão de PCV para PV (Tabela 3). Para exigências de mantença e para o cálculo das exigências dietéticas, foram utilizados os valores de perdas endógenas e

Tabela 4. Concentração de cálcio e fósforo no corpo vazio em função do peso corporal vazio.

\begin{tabular}{cccc}
\hline \multirow{2}{*}{$\begin{array}{c}\text { Peso vivo } \\
(\mathrm{kg})\end{array}$} & $\begin{array}{c}\text { Peso corporal } \\
\text { vazio } \\
(\mathrm{kg})\end{array}$ & \multicolumn{2}{c}{$\begin{array}{c}\text { Nutriente } \\
(\mathrm{g} / \mathrm{kg})\end{array}$} \\
\cline { 3 - 4 } & & Cálcio $^{(1)}$ & Fósforo $^{(2)}$ \\
\hline 15,0 & 12,9 & 15,477 & 8,739 \\
17,5 & 15,2 & 15,023 & 8,349 \\
20,0 & 17,5 & 14,644 & 8,028 \\
22,5 & 19,8 & 14,320 & 7,757 \\
25,0 & 21,2 & 14,143 & 7,117 \\
27,5 & 23,5 & 13,882 & 6,832 \\
30,0 & 25,7 & 13,658 & 6,593 \\
32,5 & 28,0 & 13,447 & 6,373 \\
35,0 & 30,2 & 13,264 & 6,184 \\
\hline
\end{tabular}

(1) Valores calculados pela equação: $\log \mathrm{Ca}=-1,06474+$ $0,818621 . \log \mathrm{PCV}$. ${ }^{(2)}$ Até o peso vivo de $22,5 \mathrm{~kg}$ de peso vivo, os valores foram calculados pela equação: $\log \mathrm{P}=-0,91433+0,721648$.log PCV; a partir de $25,0 \mathrm{~kg}$ de peso vivo, os valores foram calculados pela equação: $\log \mathrm{P}=-1,14164+0,762485 \cdot \log \mathrm{PCV}$. disponibilidade propostos pelo Agricultural Research Council (1980). As exigências líquidas e dietéticas de Ca e P para cordeiros Santa Inês são apresentadas na Tabela 6.

As exigências líquidas para o ganho de Ca obtidas neste trabalho são, aproximadamente, 5,7\% superiores às citadas pelo Agricultural Research Council (1980) para cordeiros com $15 \mathrm{~kg}$ de PV e $12 \%$ inferiores para animais com $35 \mathrm{~kg}$. O aumento da diferença nas exigências de $\mathrm{Ca}$ para o ganho entre os dois trabalhos à medida que aumenta o PV dos animais é devido ao fato de o Agricultural Research Council (1980) considerar a concentração de Ca no ganho de PV constante durante o crescimento do animal, ao passo que este trabalho considera que ocorre uma diminuição na quantidade de $\mathrm{Ca}$ por unidade de ganho de PV à medida que aumenta o peso corporal. As exigências dietéticas totais de $\mathrm{Ca}$ obtidas neste trabalho são, aproximadamente, $12 \%$ menores do que as citadas pelo National Research Council (1985), para cordeiros com $15 \mathrm{~kg}$ de PV e $31 \%$ menores para animais com $35 \mathrm{~kg}$. Com relação às normas preconizadas pelo Agricultural and Food Research Council (1991), foram 9\% e 8\% superiores para animais com 15 e $35 \mathrm{~kg}$ de PV, respectivamente, apresentando uma taxa de ganho de $200 \mathrm{~g} /$ dia.

Para animais com $15 \mathrm{~kg}$ de PV, apresentando uma taxa de ganho diário de $200 \mathrm{~g}$, verificou-se que as

Tabela 5. Composição de cálcio e fósforo do ganho em peso de corpo vazio (PCV) de cordeiros da raça Santa Inês.

\begin{tabular}{|c|c|c|}
\hline \multirow{2}{*}{$\begin{array}{c}\text { Peso corporal } \\
\text { vazio }(\mathrm{kg})\end{array}$} & \multicolumn{2}{|c|}{ Nutriente $(\mathrm{g} / \mathrm{kg})$} \\
\hline & Cálcio $^{(1)}$ & Fósforo $^{(2)}$ \\
\hline 12,5 & 12,74 & 6,36 \\
\hline 15,0 & 12,33 & 6,05 \\
\hline 17,5 & 11,99 & 5,79 \\
\hline 20,0 & 11,70 & 5,58 \\
\hline 22,5 & 11,45 & 5,09 \\
\hline 25,0 & 11,24 & 4,97 \\
\hline 27,5 & 11,04 & 4,85 \\
\hline 30,0 & 10,87 & 4,76 \\
\hline
\end{tabular}

(1) Valores calculados pela equação: $\mathrm{Y}^{\prime}=0,070524 \cdot \mathrm{PCV}^{-0,181379}$. (2) Até $20,0 \mathrm{~kg}$ de peso corporal vazio os valores foram calculados pela equação: $\mathrm{Y}^{\prime}=0,087901 . \mathrm{PCV}^{-0,278352}$; a partir de $25,0 \mathrm{~kg}$ de peso vivo, os valores foram calculados pela equação: $\mathrm{Y}^{\prime}=0,055029 \cdot \mathrm{PCV}^{-0,237514}$. 
Tabela 6. Exigências líquidas e dietéticas de cálcio e fósforo para a mantença e para o ganho em peso vivo (g/animal/dia).

\begin{tabular}{|c|c|c|c|c|c|c|c|c|}
\hline \multirow{3}{*}{$\begin{array}{c}\text { Peso vivo } \\
\text { (kg) }\end{array}$} & \multirow{2}{*}{\multicolumn{2}{|c|}{ Mantença $^{(1)}$}} & \multicolumn{6}{|c|}{ Ganho diário $(\mathrm{g})$} \\
\hline & & & \multicolumn{2}{|c|}{100} & \multicolumn{2}{|c|}{200} & \multicolumn{2}{|c|}{300} \\
\hline & $\mathrm{Ca}$ & $\mathrm{P}$ & $\mathrm{Ca}$ & $\mathrm{P}$ & $\mathrm{Ca}$ & $\mathrm{P}$ & $\mathrm{Ca}$ & $\mathrm{P}$ \\
\hline & \multicolumn{8}{|c|}{ Exigência líquida (g/animal/dia) } \\
\hline 15 & 0,240 & 0,210 & 1,163 & 0,582 & 2,326 & 1,164 & 3,489 & 1,746 \\
\hline 20 & 0,320 & 0,280 & 1,099 & 0,533 & 2,198 & 1,066 & 3,297 & 1,599 \\
\hline 25 & 0,400 & 0,350 & 1,052 & 0,499 & 2,104 & 0,998 & 3,156 & 1,497 \\
\hline 30 & 0,480 & 0,420 & 1,010 & 0,445 & 2,020 & 0,890 & 3,030 & 1,335 \\
\hline 35 & 0,560 & 0,490 & 0,982 & 0,428 & 1,964 & 0,856 & 2,946 & 1,284 \\
\hline \multicolumn{9}{|c|}{ Exigência dietética (g/animal/dia) } \\
\hline 15 & 0,353 & 0,288 & 1,710 & 0,797 & 3,420 & 1,594 & 5,130 & 2,391 \\
\hline 20 & 0,470 & 0,384 & 1,616 & 0,731 & 3,232 & 1,462 & 4,848 & 2,193 \\
\hline 25 & 0,588 & 0,479 & 1,547 & 0,684 & 3,094 & 1,368 & 4,641 & 2,052 \\
\hline 30 & 0,706 & 0,575 & 1,485 & 0,609 & 2,970 & 1,218 & 4,455 & 1,827 \\
\hline 35 & 0,823 & 0,671 & 1,444 & 0,587 & 2,888 & 1,174 & 4,332 & 1,761 \\
\hline
\end{tabular}

exigências dietéticas totais de Ca foram menores que as recomendadas por Annenkov (1982) e Okoye et al. (1980), porém semelhantes às recomendadas por Hodge (1973).

No caso do P, comparando-se as exigências líquidas obtidas neste trabalho com as do Agricultural Research Council (1980), observa-se que estas são, aproximadamente, $3 \%$ inferiores para cordeiros com $15 \mathrm{~kg}$ de PV e $40 \%$ inferiores para animais com $35 \mathrm{~kg}$.

Para animais com $35 \mathrm{~kg}$ de peso vivo e uma taxa de ganho diário de 200 g, Annenkov (1982) recomenda uma ingestão diária de P de 3,15 g; o National Research Council (1985), de 2,55 g, e o Agricultural Research Council (1980), de 2,2 g. Esses valores são, respectivamente, $68 \%, 36 \%$ e $17 \%$ maiores que os encontrados neste trabalho.

Nota-se que existe diferença entre os valores verificados neste trabalho e os valores citados pela literatura. Segundo Silva (1995), as estimativas das exigências líquidas de $\mathrm{Ca}$ e $\mathrm{P}$ de diversos trabalhos conduzidos com bovinos no Brasil também apresentaram diferenças de até $100 \%$ dos valores propostos pelo Agricultural and Food Research Council (1991).

\section{Conclusões}

1. Os valores de composição corporal de Ca variam de 15,48 a 13,26 g/kg de peso corporal vazio, e de P, de 8,74 a $6,18 \mathrm{~g} / \mathrm{kg}$ de peso corporal vazio.
2. Os requerimentos líquidos de $\mathrm{Ca}$ e $\mathrm{P}$ para o ganho em peso variam de $11,63 \mathrm{a} 9,82 \mathrm{~g} / \mathrm{kg}$ de peso vivo e de 5,82 a 4,28 g/ $\mathrm{kg}$ de peso vivo, respectivamente.

\section{Referências}

AGRICULTURAL AND FOOD RESEARCH COUNCIL (Farnham Royal, Inglaterra). A reappraisal of the calcium and phosphorus requirements of sheep and cattle. Nutrition Abstracts and Reviews, Series B, Wallingford, v. 61, n. 9, p. 573-612, 1991.

AGRICULTURAL RESEARCH COUNCIL (Farnham Royal, Inglaterra). The nutrient requirements of farm livestock. Wallingford : CAB International, 1980. 351 p.

ANNENKOV, B. N. Mineral feeding of sheep. In: GEORGIEVSKII, V. I.; ANNENKOV, B. N.; SAMOKHIN, V. I. Mineral nutrition of animals. London : Butterworths, 1982. p. 321-354.

BOIN, C. Exigências de minerais pelas categorias do rebanho bovino e funções desses nutrientes. In: SIMPÓSIO SOBRE NUTRIÇÃO DE BOVINOS, 3., 1985, Piracicaba. Anais... Piracicaba : FEALQ, 1985. p. 15-46.

BURTON, J. H.; ANDERSON, M.; REID, J. T. Some biological aspects of partial starvation: the effect of weight loss and regrowth on body composition in sheep. British Journal of Nutrition, Wallingford, v. 32, n. 3, p. 515527,1974 
BURTON, J. H.; REID, J. T. Interrelationships among energy input, body size, age and body composition of sheep. Journal of Nutrition, Bethesda, v. 97, n. 4. p. 517-524, 1969.

CARVALHO, D. R. Composição corporal e exigências nutricionais de macroelementos inorgânicos de bovinos. Viçosa : UFV, 1989. 84 p. Dissertação de Mestrado.

ESTRADA, L. H. C. Composição corporal e exigências de proteína, energia e macroelementos minerais $(\mathbf{C a}, \mathbf{P}, \mathbf{M g}$, $\mathrm{K}$ e Na): características de carcaça e desempenho do nelore e mestiço em confinamento. Viçosa: UFV, 1996. 128 p. Tese de Doutorado.

GERASEEV, L. C.; PEREZ, J. R. O.; RESENDE, K. T.; SILVA FILHO, J. C.; BONAGURIO, S. Composição corporal e exigências nutricionais em cálcio e fósforo para ganho e mantença de cordeiros Santa Inês dos $15 \mathrm{~kg}$ aos $25 \mathrm{~kg}$ de peso vivo. Revista Brasileira de Zootecnia, Viçosa, v. 29, n. 1, p. 261-268, 2000.

GRACE, N. D. Amounts and distribution of mineral elements associated with fleece-free empty body weight gains in the grazing sheep. New Zealand Journal of Agricultural Research, Wellington, v. 26, p. 59-70, 1983.

HODGE, R. W. Calcium requirements of the young lamb. II. Estimation of calcium requirements by the factorial method. Australian Journal of Agricultural Research, Collingwood, v. 24, p. 237-243, 1973.

KELLAWAY, R. C. The effects of plane nutrition, genotype and sex on growth, body composition and wool production in grazing sheep. Journal of Agricultural Science, Cambridge, Grã-Bretanha, v. 80, n. 1, p. 17-27, 1973.

LANA, R. P.; FONTES, C. A. A.; PERON, A. J.; QUEIROZ, A. C.; PAULINO, M. F.; SILVA, D. J. Composição corporal e do ganho de peso e exigências de energia, proteína e macroelementos minerais ( $\mathrm{Ca}, \mathrm{P}, \mathrm{Mg}, \mathrm{Na}$ e $\mathrm{K}$ ) de novilhos de cinco grupos raciais. 3. Conteúdo corporal e do ganho de peso e exigências de macroelementos minerais. Revista da Sociedade Brasileira de Zootecnia, Viçosa, v. 21, n. 3, p. 538-544, 1992.

LOOSLI, J. K.; GUEDES, A. C. Problemas de nutrição mineral relacionados aos climas tropicais. In: SIMPÓSIO LATINOAMERICANO SOBRE PESQUISA EM NUTRIÇÃO MINERAL DE RUMINANTES EM PASTAGENS, 1976, Belo Horizonte. Anais... Belo Horizonte : UFMG/UFV/ESAL/ EPAMIG, 1976. p. 1-10.

NATIONAL RESEARCH COUNCIL (Washington, Estados Unidos). Nutrient requirements of domestic animals: nutrient requirements of sheep. Washington, 1985. $99 \mathrm{p}$.

NOUR, A. Y. M.; THONNEY, M. L. Carcass, soft tissue and bone composition of early and late maturing steers fed two diets in two housing types and serially slaughtered a wide weight range. Journal of Agricultural Science, Cambridge, GrãBretanha, v. 109, n. 3, p. 345-355, 1987.

OKOYE, F. C.; UMUNNA, N. N.; CHINEME, C. N. Calcium and phosphorus requirements of growing yankasa lambs in the savanna region of Nigeria: estimation of calcium and phosphorus requirements by the factorial method. East African
Agricultural and Forestry Journal, Nairobi, v. 45, n. 4, p. 269-276, 1980.

PAULINO, M. F. Composição corporal e exigências de energia e macroelementos minerais $(\mathrm{Ca}, \mathrm{P}, \mathrm{Mg}$, Na e K) de bovinos não-castrados de quatro raças zebuínas em confinamento. Viçosa : UFV, 1996. 80 p. Tese de Doutorado.

PIRES, C. C.; FONTES, C. A. A.; GALVÃO, J. G.; QUEIROZ, A. C.; SILVA, J. F. C.; PAULINO, M. F. Exigências nutricionais de bovinos de corte em acabamento. III. Exigências de cálcio e fósforo para o ganho. Revista da Sociedade Brasileira de Zootecnia, Viçosa, v. 22, n. 1, p. 133-143, 1993.

RATTRAY, P. V.; GARRET, W. N.; MEYER, H. H.; BRADFORD, G. E.; EAST, N. E.; HINMAN, N. Body and carcass composition of targhee and finn-targhee lambs. Journal of Animal Science, Champaign, v. 37, n. 4, p. 892897, 1973.

RESENDE, K. T. Métodos de estimativa da composição corporal e exigências nutricionais de proteína, energia e macroelementos inorgânicos de caprinos em crescimento. Viçosa : UFV, 1989. 130 p. Tese de Doutorado.

RIBEIRO, S. D. A. Composição corporal e exigências em proteína, energia e macrominerais de caprinos mestiços em fase inicial de crescimento. Jaboticabal : UNESP-FCAVJ, 1995. 100 p. Dissertação de Mestrado.

SEARLE, T. W.; GRAHAM, N. M.; DONNELLY, J. B. The effect of plane of nutrition on the body composition of two breeds of weaner sheep fed a high protein diet. Journal of Agricultural Science, Cambridge, Grã-Bretanha, v. 98, n. 2, p. 241-245, 1982.

SEARLE, T. W.; GRAHAM, N. M.; SMITH, E. Studies of weaned lambs, before, during and after a period of weight loss. II. Body composition. Australian Journal of Agricultural Research, Collingwood, v. 30, n. 4, p. 525531, 1979.

SILVA, D. J. Análise de alimentos: métodos químicos e biológicos. Viçosa : UFV-Imprensa Universitária, 1981. $165 \mathrm{p}$.

SILVA, J. F. C. Exigências de macroelementos inorgânicos para bovinos: o sistema ARC/AFRC e a experiência no Brasil. In: SIMPÓSIO INTERNACIONAL SOBRE EXIGÊNCIAS NUTRICIONAIS DE RUMINANTES, 1995, Viçosa. Anais... Viçosa : UFV, 1995. p. 467-504.

SILVA, J. F. C. Metodologia para determinação de exigências nutricionais de ovinos. In: SILVA SOBRINHO, A. G; BATISTA, A. M. V.; SIQUEIRA, E. R.; ORTOLANI, E. L.; SUSIN, I.; SILVA, J. F. C.; TEIXEIRA, J. C.; BORBA, M. F. S. Nutrição de ovinos. Jaboticabal : FUNEP, 1996. p. 1-68.

SNEDECOR, G. W.; COCHRAN, W. G. Statistical methods. 6. ed. Ames : The Iowa State University Press, 1967. $593 \mathrm{p}$. 